\title{
DOS IMPLICANCIAS DE LA IGUALDAD RELACIONAL
}

\author{
Felipe Jiménez Castro \\ New York University
}

\begin{abstract}
RESUMEN: Una discusión central dentro del liberalismo igualitario se da entre el igualitarismo de la suerte y el igualitarismo relacional. Este artículo argumenta que, de estimarse plausible, la igualdad relacional tiene al menos dos implicancias relevantes. La primera de ellas es que la teoría y práctica políticas debieran alejarse de la pregunta sobre la desigualdad material, y acercarse a la pregunta acerca de las condiciones materiales suficientes para que los ciudadanos puedan vincularse entre sí, reconociéndose como individuos igualmente valiosos, sin relaciones de dominación interpersonal. La segunda implicancia consiste en que la creciente atención filosófica dedicada a la igualdad relacional debería llevarnos a la reflexión filosófica acerca del derecho privado, en el que las interacciones entre individuos juegan un rol crucial.

Palabras Clave: liberalismo igualitario, igualitarismo de la suerte, igualitarismo relacional, teoría del derecho privado, suficientarismo.

RECIBIDO: octubre 2016; ACEPTADO: noviembre 2016.
\end{abstract}

Felipe Jiménez Castro. Abogado de la Pontificia Universidad Católica de Chile. Magíster en teoría del derecho y estudiante de JSD en la New York University Law School. Email: felipe.jimenezcastro@law.nyu.edu.

* El autor quiere señalar: "Una primera versión de este trabajo fue desarrollada en el contexto del seminario de teoría política del profesor Wojciech Sadurski, en el semestre de primavera de 2016, en la Facultad de Derecho de NYU. Agradezco a Javier Gallego, Crescente Molina y Olof Page por los comentarios que formularon a una versión anterior de este trabajo, así como a Hanoch Dagan, Liam Murphy y Marcela Prieto por varias conversaciones vinculadas a los problemas tratados en él. Asimismo, agradezco los valiosos comentarios de los árbitros anónimos que evaluaron este trabajo para Estudios Públicos". 


\section{TWO IMPLICATIONS OF RELATIONAL EQUALITY}

ABSTRACT: A central discussion within liberal egalitarianism is that between luck egalitarians and relational egalitarians. This paper claims that, if plausible, the idea of relational equality can have at least two important implications. The first of these implications is that political theory and practice should move away from the question of material inequality as such, and towards the question about what material conditions are sufficient for citizens to relate to each other as equally valuable individuals, without any interpersonal relationships of domination. The second implication is that the growing philosophical attention towards relational equality should also lead us towards philosophical reflection about private law, an area in which private interactions between individuals are central.

Keywords: liberal egalitarianism, luck egalitarianism, relational egalitarianism, private law theory, sufficientarianism.

RECEIVED: October 2016; ACCEPTED: November 2016.

\section{INTRODUCCIÓN}

E n las primeras páginas de Teoría de la justicia, John Rawls escribía que la justicia es la primera virtud de las instituciones sociales. ${ }^{1}$ La teoría de la justicia de Rawls y su liberalismo político han jugado un papel crucial en parte importante de las discusiones de la filosofía política de los últimos cuarenta años, ${ }^{2}$ particularmente dentro del liberalismo igualitario. Los liberales igualitarios de las últimas décadas han trabajado, fundamentalmente, dentro del marco de la teoría rawlsiana o en contraposición a ella. Y la mayoría de ellos, al margen de sus diferencias, han estado en general escasamente interesados en los aspectos no distributivos de la justicia; para el liberalismo igualitario, pues, la primacía de la justicia ha sido la primacía de la justicia distributiva. ${ }^{3}$

${ }^{1}$ John Rawls, A Theory of Justice (Cambridge: Harvard University Press, 1971), 3 .

${ }^{2}$ Sólo unos años después de Teoría de la justicia, Robert Nozick hacía ver que los filósofos políticos, después de Rawls, tenían que trabajar dentro del marco de su teoría o explicar por qué no lo hacían. Robert Nozick, Anarchy, State, and Utopia (New York: Blackwell, 1974), 183.

${ }^{3}$ Como Samuel Scheffler lo plantea lúcidamente: "La justicia distributiva ha sido una preocupación tan central de la filosofía política reciente, que ha tendido a desplazar otros temas importantes. Sin reconocerlo explícitamente, muchos de 
Este trabajo tiene como propósito argumentar que una de las discusiones centrales al interior del liberalismo igualitario - el desacuerdo entre igualitarismo de la suerte y el igualitarismo relacional- puede tener implicancias relevantes, precisamente en relación a aspectos no estrictamente distributivos de la justicia. ${ }^{4}$ El objetivo del trabajo, entonces, no es el de zanjar definitivamente el desacuerdo entre igualitaristas de la suerte e igualitaristas relacionales, sino que destacar algunas implicancias poco exploradas que el ideal de igualdad relacional, si se estima plausible, puede tener para la filosofía política.

A lo largo de este trabajo, me refiero a la idea de igualdad o igualitarismo relacional, sin perjuicio de que las etiquetas igualitarismo democrático o igualitarismo social podrían cumplir el mismo rol. Todas ellas se refieren - como se verá en lo sucesivo - a las corrientes igualitaristas que, en contraposición al llamado igualitarismo de la suerte, juzgan la justicia de una situación no por el patrón de distribución material que la caracteriza, sino que por la calidad moral y política de las relaciones interpersonales que ella genera. ${ }^{5}$

Una primera implicancia del ideal de la igualdad relacional, según argumenta este trabajo, es que - en la medida en que la igualdad relacional sea estimada como un ideal político valioso - la teoría y la práctica política no deberían centrarse en la cuestión de la desigualdad material como tal, sino en la pregunta, más prioritaria, acerca de las condiciones materiales que permiten que cada individuo lleve una vida consistente con la igualdad relacional. En este aspecto, el trabajo sigue de cerca el planteamiento de autores como Elizabeth Anderson, que

nosotros hemos aceptado implícitamente un paradigma distributivista, que trata a la justicia distributiva como una categoría normativa predominante que tiene igual autoridad regulativa sobre todas las instituciones sociales, incluyendo aquellas vinculadas a los contratos y la responsabilidad extracontractual". Samuel Scheffler, "Distributive Justice, the Basic Structure and the Place of Private Law", Oxford Journal of Legal Studies 35 (2015): 229. La traducción es propia.

${ }^{4}$ Como destaca Wolff, las cuestiones distributivas, aunque importantes, no agotan las preocupaciones de una perspectiva igualitarista. Jonathan Wolff, "Social Equality and Social Inequality", en Social Equality, ed. Carina Fourie, Fabian Schuppert \& Ivo Wallimann-Helmer (Oxford: Oxford University Press, 2015), 211.

${ }^{5}$ En la utilización de los tres pares de etiquetas como sinónimos, sigo a Daniel Loewe, "Refugiados climáticos: ¿Quién debe cargar los costos?", Revista Interdisciplinar Da Mobilidade Humana 22 (2014): 177, nota 17. 
defienden la vinculación entre igualdad relacional y suficiencia, ${ }^{6}$ pero intenta proponer un argumento más robusto y sólido frente a las críticas tradicionales al suficientarismo. De acuerdo a tal argumento, la preocupación política fundamental no debiera ser la de asegurar un patrón determinado de distribución de bienes, sino la de asegurar que cada persona cuente con bienes suficientes. Este trabajo, entonces, defiende una conexión entre el igualitarismo relacional y el suficientarismo. Dada esa conexión, un Estado enfocado en asegurar la igualdad relacional no debiera ocuparse de disminuir la desigualdad material, sino la pobreza (entendida como las condiciones materiales bajo las cuales los individuos no son capaces de llevar vidas no dominadas o como ciudadanos iguales), la cual — como señala Frankfurt- "es un impedimento serio y moralmente objetable a una buena vida". ${ }^{7}$ Sin embargo, y dado el hecho económico de la escasez de bienes, el criterio de suficiencia aquí defendido puede requerir importantes medidas redistributivas.

La segunda implicancia que este trabajo explora se aleja de las disputas internas de la filosofía política igualitarista y se acerca más a las instituciones jurídicas concretas, en particular al derecho privado. En este aspecto, este trabajo argumenta que la atención a la (des)igualdad en las relaciones interpersonales entre ciudadanos - cuestión en la que el igualitarismo relacional ha puesto el foco- debería hacernos volver a la pregunta acerca de la justicia en las interacciones privadas entre individuos. Esto implica que la creciente atención filosófica respecto a la igualdad relacional debiera también llevarnos a reflexionar críticamente acerca del derecho privado - en particular, los contratos, la responsabilidad por daños y el enriquecimiento injustificado-,${ }^{8}$ en el cual las relaciones interpersonales ocupan un lugar central. La igualdad relacional trae como consecuencia, entonces, el retomar la reflexión filosófica y política acerca de las instituciones de derecho privado, una reflexión históricamente conectada a la idea de justicia correctiva.

${ }^{6}$ Véase, principalmente, Elizabeth Anderson, “¿Cuál es el punto de la igualdad?", en Igualitarismo: Una discusión necesaria, ed. Javier Gallego y Thomas Bullemore (Santiago: Centro de Estudios Públicos, 2016), 45-104.

${ }^{7}$ Harry Frankfurt, "Equality and Respect", Social Research 64, n. ${ }^{\circ} 1$ (1997): 5. La traducción es propia.

${ }^{8}$ Omito el derecho de bienes dado que el derecho de propiedad, a diferencia del resto del derecho privado, ha sido una (si no la) institución que ha preocupado permanentemente a los teóricos políticos centrados en problemas de justicia distributiva. 
Con el objetivo de explorar estas dos implicancias y argumentar cómo ellas emanan del ideal de igualdad relacional, el trabajo tiene la siguiente estructura. La primera parte presenta una descripción básica del desacuerdo entre el igualitarismo de la suerte y el igualitarismo relacional, y explica por qué este último es al menos plausible como interpretación del valor de la igualdad. De ahí en adelante, el argumento opera sobre la base de esa plausibilidad, a partir de la cual las partes segunda y tercera exploran dos implicancias de la igualdad relacional. La segunda parte analiza las implicancias que la igualdad relacional tiene para los desacuerdos que se suelen presentar respecto a la redistribución de la riqueza y el rol del Estado en ella. Así, la igualdad relacional debiera alejarnos de una preocupación centrada en la desigualdad material, y movernos hacia un enfoque suficientarista centrado en el aseguramiento de las condiciones materiales que permiten el desarrollo de vidas no dominadas, ${ }^{9}$ consistentes con la igualdad moral de las personas. ${ }^{10}$ La tercera parte, en tanto, se refiere a la conexión entre el igualitarismo relacional y el redescubrimiento del derecho privado como objeto de reflexión filosófico-política. La cuarta parte concluye.

${ }^{9}$ En este trabajo, entiendo por dominación la sujeción al poder o autoridad arbitrario o discrecional de otro. Desde esta perspectiva, vivir bajo relaciones de dominación consiste en vivir bajo relaciones en las cuales una de las partes está sujeta al poder arbitrario o discrecional de la otra. Sobre la idea de no dominación se ha escrito extensamente, sobre todo como parte de concepciones políticas republicanas. Al respecto, véase Frank Lovett, "Republicanism", en The Stanford Encyclopedia of Philosophy (2016), http://plato.stanford.edu/archives/spr2016/entries/republicanism/; Philip Pettit, Republicanism (Oxford: Oxford University Press, 1999); Ian Shapiro, "On Non-domination", University of Toronto Law Journal 62, n. 3 (2012): 293-336. Por cierto, no soy el primero en notar la conexión entre el ideal de la igualdad y la no dominación. En este mismo sentido, por ejemplo, véase el análisis de Thomas Scanlon, "When Does Equality Matter?" (2005): 9, https:// www.law.yale.edu/system/files/documents/pdf/Intellectual_Life/ltw-Scanlon.pdf.

${ }^{10}$ Preguntar acerca de dónde deriva la igualdad moral de las personas y por qué deben ser tratadas como iguales es fundamental, por supuesto, pero ello excede con creces el objetivo de este trabajo. En efecto, el argumento presentado aquí asume esa igualdad moral y su fundamentación, y se enmarca en consecuencia dentro de la discusión acerca de qué es lo que la igualdad moral, como quiera que sea justificada, exige. Respecto a estas preguntas fundamentales, en las que no me centro, véase Ian Carter, "El respeto y la base de la igualdad", en Igualitarismo: Una discusión necesaria, 193-228. Una estrategia similar, en el sentido de asumir — sin defender - la idea de igualdad moral de las personas, es empleada por Scanlon, "When Does Equality Matter?", 2. 


\section{EL DESACUERDO ENTRE IGUALITARISMO DE LA SUERTE E IGUALITARISMO RELACIONAL}

El desacuerdo entre el igualitarismo de la suerte y el igualitarismo relacional puede ser leído como un desacuerdo acerca de cuál es la mejor interpretación del valor de la igualdad. ${ }^{11} \mathrm{El}$ igualitarismo de la suerte considera que la igualdad consiste en la necesidad de velar por una distribución de recursos, riqueza o bienes primarios ${ }^{12}$ que sea sensible a la elección personal ${ }^{13}$ e insensible a la suerte bruta (en oposición a la suerte de opción $)^{14} \mathrm{o}$ a otras contingencias moralmente arbitrarias, tales como el talento o la habilidad innatos. ${ }^{15} \mathrm{El}$ igualitarismo de la suerte, entonces, se enfoca principalmente en la distribución material, y en su vinculación con la responsabilidad individual, para evaluar la justicia de

${ }^{11}$ Por supuesto, esto es una simplificación. Para una exposición detallada del desacuerdo, véase Elizabeth Anderson, "The Fundamental Disagreement between Luck Egalitarians and Relational Egalitarians", Canadian Journal of Philosophy 40 (2010): 1-3. En la caracterización del desacuerdo como un conflicto entre interpretaciones rivales de la igualdad, sigo a Samuel Scheffler, "The Practice of Equality", en Social Equality, ed. Carina Fourie, Fabian Schuppert e Ivo Wallimann-Helmer (Oxford: Oxford University Press, 2015), 21.

${ }^{12} \mathrm{~A}$ lo largo de este trabajo hablo indistintamente de bienes, recursos, oportunidades, etcétera. Esto se debe a que entre los teóricos del igualitarismo de la suerte no existe completo acuerdo respecto a qué ventajas son las que deben distribuirse conforme a los criterios del igualitarismo de la suerte. Al respecto, véase Javier Gallego y Thomas Bullemore, "Introducción", en Igualitarismo: Una discusión necesaria, 26-30; Carl Knight, "Luck Egalitarianism", Philosophy Compass 8, n. ${ }^{\circ}$ 10 (2013): 927-928; Samuel Scheffler, "What Is Egalitarianism?", Philosophy \& Public Affairs 31, n. ${ }^{\circ} 1$ (2003): 13.

${ }^{13}$ Respecto a los problemas que genera la sensibilidad a la elección personal, véase Scheffler, "What Is Egalitarianism?", 18; Seana Shiffrin, "Egalitarianism, Choice-Sensitivity, and Accommodation", en Reason and Value: Themes from the Work of Joseph Raz, ed. Philip Pettit (Oxford: Oxford University Press, 2004), 270-302.

${ }^{14} \mathrm{La}$ suerte de opción es la que sigue de una elección que el individuo ha tomado (por ejemplo, apostar o invertir en la bolsa), mientras que la suerte bruta es la que es enteramente independiente de la elección individual. La distinción es de Ronald Dworkin, "What is Equality? Part 2: Equality of Resources", Philosophy \& Public Affairs 10, n. 4 (1981): 283-345. Véase también Knight, "Luck Egalitarianism”, 925.

${ }^{15}$ Véase, por ejemplo, G.A. Cohen, "On the Currency of Egalitarian Justice", Ethics 99, n. 4 (1989): 906-944; Ronald Dworkin, Sovereign Virtue: The Theory and Practice of Equality (Cambridge: Harvard University Press, 2002), 287. Véase también Wojciech Sadurski, Equality and Legitimacy (Oxford: Oxford University Press, 2008), 207-214. 
una sociedad. Por esa vinculación entre distribución y responsabilidad individual, se ha dicho con razón que se trata de un proyecto conciliatorio, que se aleja de un igualitarismo estricto. ${ }^{16}$

Contra el igualitarismo de la suerte, el igualitarismo relacional postula que, ante todo, la igualdad no es un ideal de distribución material dirigido a compensar la mala suerte individual, sino que un ideal regulativo respecto de la calidad de las relaciones humanas, las que deben basarse en el presupuesto de que las vidas de todos los individuos son igualmente importantes y cada miembro de la sociedad tiene igual valor moral. ${ }^{17}$ Para el igualitarismo relacional, pues, el ideal igualitario es un ideal de relaciones en las que los ciudadanos se vinculan entre sí reconociéndose como individuos igualmente valiosos, y en las que no existe dominación interpersonal. ${ }^{18}$ Por esta razón, para esta perspectiva las relaciones privadas de dominación son inaceptables, incluso si las partes acceden voluntariamente a ellas. ${ }^{19}$

De ahí se sigue que - para los igualitaristas relacionales - el punto de vista relevante, cuando se trata de evaluar la justicia de una sociedad, no es el de la distribución material, sino el de una concepción evaluativa de las relaciones humanas y del diseño de las instituciones políticas. ${ }^{20}$ Por ello, la pregunta fundamental no es cuál distribución de bienes, recursos u oportunidades es exigible al Estado, sino que la pregunta acerca de qué tipo de relaciones y arreglos sociales permite que los individuos se relacionen como iguales. ${ }^{21}$

Dados los propósitos y la extensión de este trabajo, no pretendo zanjar definitivamente el desacuerdo entre estas dos concepciones igualitaristas. Sin embargo, a mi juicio la interpretación del igualitarismo relacional es al menos plausible como una concepción acerca del valor que normalmente le asignamos a la igualdad, y como un ideal regulativo de la acción política y el diseño institucional. Esto es así por al menos cuatro razones.

En primer término, muchas políticas de inspiración igualitarista son insensibles a la elección individual, asegurando recursos y benefi-

${ }^{16}$ En este sentido, ver Olof Page, "Igualdad, suerte y responsabilidad", en Igualitarismo: Una discusión necesaria, 236.

${ }^{17}$ Scheffler, "What Is Egalitarianism?", 33-34.

${ }^{18}$ Anderson, “¿Cuál es el punto de la igualdad?”, 46-48.

${ }^{19}$ Ibídem, 78.

${ }^{20}$ Scheffler, “What Is Egalitarianism?", 31.

${ }^{21}$ Samuel Scheffler, "Equality as the Virtue of Sovereigns: A Reply to Ronald Dworkin", Philosophy \& Public Affairs 31, n. ${ }^{\circ} 2$ (2003): 204. 
cios a personas que, encontrándose en circunstancias particularmente negativas y bajo un cierto umbral mínimo, son, sin embargo, responsables de esas condiciones en un sentido moralmente relevante. ${ }^{22}$ Esas políticas sólo pueden ser conciliadas con un igualitarismo no paternalista, a mi juicio, si entendemos el igualitarismo como algo más que el ideal distributivo del igualitarismo de la suerte.

En segundo lugar, la observación del igualitarismo relacional respecto a que hay una dimensión de la igualdad que no puede ser reducida a cuestiones puramente distributivas, sino que se vincula a la evaluación moral y política de las relaciones interpersonales, es, a mi juicio, correcta. En efecto, las relaciones que expresan, aun a un nivel simbólico, diferencias de estatus o de rango entre las personas parecen problemáticas incluso si ellas no producen un efecto negativo desde el punto de vista de la distribución material, y todavía si ellas no se producen como consecuencia de esa distribución. Es decir, la igualdad tiene una dimensión que es enteramente independiente de cuestiones distributivas. Un ejemplo claro es dado por el clasismo. El clasismo, en sus manifestaciones más dañinas, afecta la distribución material de manera evidente (por ejemplo, cuando la discriminación por origen socioeconómico afecta las oportunidades laborales de una persona). Sin embargo, el clasismo que no afecta la distribución material es aun así problemático, y produce precisamente el tipo de relaciones interpersonales que al igualitarista deberían parecerle dignas de reproche. ${ }^{23}$

En tercer término, la crítica de Elizabeth Anderson, en el sentido de que el igualitarismo de la suerte no muestra suficiente respeto a los ciudadanos, parece correcta. El igualitarismo de la suerte invita a hacer

${ }^{22}$ Un intento de conciliación entre la sensibilidad a la elección y la protección de los llamados "undeserving poor", es el de Richard J. Arneson, "Egalitarianism and the Undeserving Poor", Journal of Political Philosophy 5, n. 4 (1997): 327350. Una defensa radical de la protección de quienes parecieran no merecer protección, en la forma de un ingreso universal garantizado, es otorgada por Philippe Van Parijs, "Why Surfers Should be Fed: The Liberal Case for an Unconditional Basic Income", Philosophy \& Public Affairs 20, n. 2 (1991): 101-131.

${ }^{23}$ Por ejemplo, la figura del siútico en la historia chilena puede ser visto, plausiblemente, como un producto del clasismo. Sin embargo, el uso de esa figura no pareciera afectar la distribución material, ni tampoco tener su origen en déficits distributivos. Aun así, creo que es razonable sostener que el calificativo, desde el punto de vista igualitarista, es problemático. Respecto a la figura del siútico en la historia chilena, véase el trabajo de Óscar Contardo, Siútico: Arribismo, abajismo y vida social en Chile (Santiago: Vergara, 2008). 
juicios de carácter moralista acerca de la responsabilidad individual de las personas (por ejemplo, ¿carece la persona A de recursos porque no tiene talento o porque es floja?), que son inconsistentes con el respeto que ellas se merecen. ${ }^{24}$

Por último, el igualitarismo de la suerte se basa en una distinción entre elección y suerte que, por una parte, no es lo suficientemente robusta para cargar con el peso normativo que se le atribuye, y que, por la otra, no tiene implicancias morales atractivas. En efecto, normalmente estimamos justo que la gente sea compensada en proporción a su talento o su disposición al trabajo, pese a que estas características no son elegidas. Respecto al segundo punto, en tanto, normalmente nos parecería injusto que alguien no reciba atención médica gratuita y termine muriendo, pese a que padece una condición que se debe enteramente a la elección individual. ${ }^{25}$

Por estas razones, el igualitarismo relacional es al menos plausible frente al igualitarismo de la suerte. Dada esa plausibilidad, el argumento de este trabajo explora dos implicancias del ideal de la igualdad relacional y que, de estimarse ella plausible, son filosófica y políticamente relevantes. Para efectos del argumento, por lo demás, es indiferente si dicha plausibilidad es interpretada en un sentido fuerte, de manera que la igualdad relacional pasa a sustituir totalmente la preocupación distributiva del igualitarismo de la suerte, o en un sentido débil, de manera que la igualdad relacional es un correctivo a un enfoque demasiado estrechamente centrado en problemas de distribución material (y su vinculación con la elección individual y la compensación de la suerte bruta), ${ }^{26}$ pero que es de todas formas compatible con nociones de justicia distributiva igualitaria.

${ }^{24} \mathrm{Al}$ respecto, Anderson, “¿Cuál es el punto de la igualdad?”, 72-73; Facundo García Valverde, "La prioridad del igualitarismo democrático", Revista de Filosofía 41, n. ${ }^{\circ} 1$ (2016): 82.

${ }^{25}$ En este sentido, Anderson, “¿Cuál es el punto de la igualdad?”, 55-61; Scheffler, "What Is Egalitarianism?", 18-19.

${ }^{26}$ Por ejemplo, uno podría decir — como muchos igualitaristas de la suerteque el igualitarismo de la suerte debe ser compatible con una red de protección que resguarde a las personas, incluso frente a sus propias decisiones irresponsables, y que la mejor justificación de esa red de protección es de carácter igualitarista relacional. Respecto a la necesidad de compatibilizar el igualitarismo de la suerte con una red de protección (aunque su justificación para ello es más bien de carácter paternalista), véase Sadurski, Equality and Legitimacy, 203. 


\section{DE LA IGUALDAD RELACIONAL AL SUFICIENTARISMO27}

En primer término, la valoración de la igualdad relacional como valor político debiera llevarnos a reconsiderar la plausibilidad de argumentos suficientaristas. ${ }^{28}$ Como señala García Valverde en relación con la postura de Elizabeth Anderson, para el igualitarismo relacional "cada ciudadano tiene un derecho a las capacidades requeridas para evitar relaciones sociales opresivas y de explotación y para vivir como un ser humano capaz de perseguir sus propias concepciones del bien y participar de la vida económica, social y política de la vida de sus comunidades". ${ }^{29}$ El igualitarismo relacional, entonces, otorga apoyo al suficientarismo (en lugar de competir con él, como podría pensarse). ${ }^{30}$ Se trata, en todo caso, de un suficientarismo exigente, como se verá en lo sucesivo.

Desde ya, cabe poner de manifiesto que el argumento que se defiende en este trabajo es pluralista. El suficientarismo es defendido no como un ideal exclusivo y excluyente en materia de justicia económica y política, sino como un componente relevante de las mismas. Este enfoque pluralista, entonces, integra dentro del suficientarismo - como se verá más adelante- distintas preocupaciones normativas, y deja espacio para otras preocupaciones al margen del criterio de suficiencia. ${ }^{31}$ Como tal, esta versión limitada y pluralista del suficientarismo no

${ }^{27}$ Como he señalado antes, esta sección, más que proponer una implicancia totalmente inexplorada del igualitarismo relacional, se basa en la vinculación que ya algunos han construido entre éste y el suficientarismo, intentando ofrecer una versión más elaborada y robusta de dicha vinculación.

${ }^{28}$ Como plantea Arneson al analizar la igualdad relacional o democrática (aunque para finalmente rechazarla): "El ideal de la igualdad democrática, construido en términos amplios, es el de una sociedad en la cual personas libres interactúan como iguales (...). El elemento de suficiencia de la igualdad democrática podría estar subordinado a este ideal de igualdad social: los arreglos sociales y económicos suficientemente buenos son aquellos que sostienen la igualdad social". Richard J. Arneson, "Democratic Equality and Relating as Equals", Canadian Journal of Philosophy 40 (2010): 28-29. La traducción es propia.

${ }^{29}$ García Valverde, "La prioridad", 83.

${ }^{30}$ Muchos igualitaristas han construido la vinculación inversa: razones suficientaristas en apoyo de principios igualitaristas. Al respecto, véase Liam Shields, “The Prospects for Sufficientarianism", Utilitas 24, n. 1 (2012): 115.

${ }^{31}$ Tras la crítica del igualitarismo relacional, varios igualitaristas de la suerte han adoptado también una perspectiva pluralista. Al respecto, Knight, "Luck Egalitarianism", 930-32. 
presenta los problemas que otras concepciones suficientaristas, particularmente cuando niegan la relevancia del igualitarismo, sí padecen. ${ }^{32}$ En este sentido, y al igual que en otros ámbitos de la filosofía política y en relación con otros valores políticos, a mi juicio cualquier defensa monista de la noción de suficiencia, de acuerdo a la cual la suficiencia sería lo único que importa, está destinada al fracaso. Una defensa completa del pluralismo de valores, por supuesto, excede los propósitos de este trabajo. ${ }^{33} \mathrm{Al}$ respecto, simplemente asumo como cierto que una perspectiva monista de los valores políticos presenta problemas graves. Así, por ejemplo, una visión monista empobrece nuestra comprensión de la vida moral y es reductiva. ${ }^{34}$ Para el pluralismo que asumo, por el contrario, debemos reconocer que existe una diversidad de valores morales que, a veces, entran en conflicto entre sí. ${ }^{35}$ Este pluralismo, a mi juicio, es la perspectiva más consistente con la experiencia moral cotidiana de los seres humanos. ${ }^{36}$

En relación con el suficientarismo, de todas formas, el punto de partida del argumento de esta sección es que, aunque no sea un ideal distributivo en sí mismo, la igualdad relacional tiene implicancias distributivas. ${ }^{37}$ Esas implicancias, sin embargo, se alejan del enfoque centrado en una distribución determinada, o en la conexión entre esa distribución y la suerte y la responsabilidad individual. Por el contrario, tales implicancias llevan a la revalorización de la teoría y la acción políticas dirigidas a asegurar condiciones materiales consistentes con la igualdad moral de las personas. La igualdad relacional no requiere, ne-

${ }^{32} \mathrm{Al}$ respecto, véase Paula Casal, "Por qué la suficiencia no basta", en Igualitarismo: Una discusión necesaria, 269-301.

${ }^{33}$ Dicha defensa fue, tal vez, el proyecto central de la trayectoria intelectual de Isaiah Berlin. Para Berlin, la creencia de que se podía encontrar una fórmula que unificara todos los fines de los seres humanos era una ilusión cuya falsedad se puede demostrar. Los propósitos de los seres humanos son múltiples e incompatibles. La tragedia derivada de la necesidad de elegir entre esos fines incompatibles, para Berlin, era simplemente parte de la condición humana. Isaiah Berlin, Liberty, ed. Henry Hardy (Oxford: Oxford University Press, 2002), 214.

${ }^{34}$ Elizabeth Anderson, Value in Ethics and Economics (Cambridge: Harvard University Press, 1995), 5.

${ }^{35}$ Gregory S. Alexander, "Pluralism and Property", Fordham Law Review 80 (2011): 1020 .

${ }^{36}$ Ibídem, 1035.

${ }^{37}$ David Miller, "Equality and Justice", Ratio 10, n. ${ }^{\circ} 3$ (1997): 234. En el mismo sentido, Scheffler, "The Practice of Equality", 21. 
cesariamente, la igualación de un determinado objeto (como el poder, el prestigio o la riqueza), sino que exige que cualesquiera diferencias que se produzcan sean consistentes con relaciones interpersonales en las que no existe la opresión, las jerarquías sociales o la dominación, y en las que los vínculos personales no son el producto de las diferencias de clase. ${ }^{38}$ La suficiencia designa, así, al conjunto de condiciones materiales que permiten tales relaciones.

Esta idea se podría objetar en razón de que la conexión entre suficiencia y relaciones no dominadas es inconsistente, puesto que pueden existir relaciones opresivas aun en circunstancias en que ambas partes se encuentren por sobre el umbral de suficiencia. ${ }^{39} \mathrm{Si}$ ello es así, dice la objeción, entonces la suficiencia es consistente con la dominación. Sin embargo, esta crítica asume como punto de partida justamente lo que yo niego: que alguien pueda tener lo suficiente y, pese a eso, vivir relaciones de dominación. Por el contrario, si — como sugiere mi argumentoel estándar de suficiencia es visto como la consecuencia de un ideal (el de la igualdad relacional) de vínculos interpersonales en los que no existe dominación, la posibilidad de relaciones en que ambas partes están por sobre el umbral de suficiencia pero existe dominación está excluida por definición. En efecto, bajo el argumento defendido aquí, precisamente, el ideal de relaciones interpersonales no dominadas dota de contenido al criterio de suficiencia: cuánto sea lo suficiente depende de cuál umbral es el necesario para evitar la dominación.

De acuerdo a los argumentos suficientaristas tradicionales, que suelen postularse en contraposición al igualitarismo, el Estado no debería tener como foco alcanzar la igualdad económica o una determinada distribución ideal de bienes o riqueza, sino que reducir (e idealmente eliminar) la pobreza. Para Frankfurt, por ejemplo, lo relevante respecto a la distribución de recursos económicos no es que todos tengan lo mismo, sino que todos tengan lo suficiente. ${ }^{40}$ En términos más positivos, la preocupación central no debería ser redistribuir riqueza desde ciertos bolsillos hacia otros, sino que asegurar las condiciones materiales que permitan que cada individuo cuente con recursos suficientes para llevar

\footnotetext{
${ }^{38}$ Miller, "Equality and Justice", 232.

${ }^{39}$ Agradezco a Olof Page por sugerirme esta posible objeción.

${ }^{40}$ Harry Frankfurt, "Equality as a Moral Ideal”, Ethics 98, n. ${ }^{\circ} 1$ (1987): 21.
} 
a cabo una vida humana decente y digna, ${ }^{41}$ que sea consistente con la igualdad moral de todos los seres humanos, ${ }^{42}$ y que les permita -en definitiva - vivir como agentes morales responsables. ${ }^{43}$

Sin embargo, y en contraste con esta defensa tradicional del suficientarismo en la literatura filosófica, a partir de la cual el suficientarismo es esgrimido como una alternativa al igualitarismo, ${ }^{44}$ el argumento de este trabajo ve al suficientarismo como la conclusión lógica del igualitarismo democrático. ${ }^{45}$ No me parece correcta, entonces, la contradicción que podría verse a priori entre suficientarismo e igualitarismo. Por el contrario, a mi juicio la mejor versión del suficientarismo define lo suficiente precisamente al ideal de la igualdad relacional. Por ello, tal como aquí se defiende, el suficientarismo es también abierto, en términos de admitir otras exigencias no basadas en la suficiencia que derivan del ideal de la igualdad relacional. Pese a ello, la igualdad relacional reclama cierta prioridad y urgencia para las demandas de suficiencia antes que las de distribución igualitaria.

La contraposición entre un enfoque centrado en la suficiencia y uno centrado en la igualdad distributiva podría ser vista como un eje

${ }^{41}$ Como Olof Page me ha sugerido, al revisar una versión anterior de este trabajo, el objetivo de asegurar tales condiciones también puede implicar la necesidad de redistribuir riqueza desde ciertos bolsillos hacia otros. Eso puede ser correcto, pero es enteramente contingente, y depende — entre otras cosas - de la idoneidad instrumental, evaluada concretamente, de las medidas redistributivas. Esto significa que un suficientarismo igualitario, como el que defiende este trabajo, está abierto a la posibilidad de que, en ciertas circunstancias, la redistribución sea instrumentalmente necesaria. Pero el suficientarismo es compatible - de una manera en que el igualitarismo de la suerte y, en general, el igualitarismo distributivo, no lo es- con que otros medios resulten más idóneos. Como se verá más adelante, a mi juicio, en definitiva, la respuesta a la pregunta de si debemos redistribuir o no es enteramente contingente, y depende de cuestiones empíricas concretas que la filosofía moral no está en condiciones de analizar.

${ }^{42}$ La version clásica del argumento está en Frankfurt, "Equality as a Moral Ideal"; Frankfurt, "Equality and Respect". Véase también Roger Crisp, "Equality, Priority, and Compassion", Ethics 113, n. ${ }^{\circ} 4$ (2003): 745-763. En un sentido similar, basado en la idea de un umbral mínimo de capacidades consistente con la dignidad humana, Martha C. Nussbaum, Frontiers of Justice: Disability, Nationality, Species Membership (Cambridge: Harvard University Press, 2009), 70-71.

${ }^{43}$ En este sentido, Violetta Igneski, "Equality, Sufficiency, and the State", Dialogue: Canadian Philosophical Review 46, n. ${ }^{\circ} 2$ (2007): 311-334.

${ }^{44}$ Particularmente, Frankfurt, "Equality as a Moral Ideal"; Frankfurt, "Equality and Respect".

${ }^{45}$ En el mismo sentido, Igneski, "Equality, Sufficiency”, 315. 
central de la discusión política en la mayoría de los países occidentales. Algunas posturas, menos igualitaristas, bajo esta lectura, tenderían a promover la idea de que el Estado debería luchar contra la pobreza, asegurando que ningún individuo caiga bajo un umbral mínimo -en términos de ingresos, acceso a bienes y servicios básicos, etcétera-, por sobre el cual la libre competencia y la meritocracia deberían determinar la distribución. Otras concepciones más igualitaristas, en cambio, tenderían a argumentar que este criterio de suficiencia no basta, y que el Estado debería asegurar una distribución justa de los recursos producidos por la cooperación social. Para una concepción de este tipo, las diferencias en ingreso, riqueza y recursos deben ser justificadas, ${ }^{46}$ ya que el punto de vista relevante no es asegurar un mínimo, sino que asegurar lo que es justo.

Esta forma de interpretar los desacuerdos políticos es ciertamente atractiva, y puede capturar parte de la oposición entre concepciones políticas divergentes. Sin embargo, si la igualdad relacional es un ideal atractivo para el liberalismo igualitario, ella puede proveer un camino valioso hacia un diálogo político más productivo y fértil entre distintas concepciones. En efecto, si adoptamos la perspectiva relacional, la oposición entre concepciones políticas suficientaristas e igualitaristas puede ser reinterpretada como una discrepancia entre distintas concepciones respecto a qué es lo suficiente para asegurar que los ciudadanos se vinculen como iguales, sin que nadie sufra ni ejerza formas de dominación. Las distintas posturas seguirán estando en desacuerdo, por supuesto, ya que el criterio de suficiencia para una será probablemente menos demandante que para la otra (que, después de todo, vería la suficiencia como una demanda basada en un igualitarismo exigente). ${ }^{47}$ Por lo demás, dada la escasez de bienes, las concepciones comprometidas con un estándar exigente de suficiencia seguirán, como práctica política, requiriendo que el Estado redistribuya significativamente recursos. Pero bajo esta interpretación, el desacuerdo es más transparente que bajo la

${ }^{46}$ En el caso del principio de diferencia de Rawls, tal justificación está dada por la necesidad de las diferencias para mejorar las condiciones de los menos aventajados. Véase Rawls, A Theory of Justice, 75-83.

${ }^{47}$ Como se podrá anticipar, y explicaré más detalladamente a continuación, a mi juicio el suficientarismo y el igualitarismo no son concepciones necesariamente rivales. Por el contrario, el igualitarismo - al menos en el sentido relacional— da un fundamento a la idea de suficiencia. 
tradicional, que ve a las concepciones políticas opuestas preocupadas de preguntas distintas. El desacuerdo, finalmente, se produce entre facciones que hablan el mismo lenguaje.

Más allá del aporte del suficientarismo basado en la igualdad relacional al diálogo político, este argumento también tiene implicancias relevantes para la teoría y la acción política de inspiración igualitarista. Así, bajo el argumento suficientarista al que, como he señalado, debe llevar el igualitarismo relacional, la igualdad no es un resultado material que tratamos de obtener, sino que el punto de partida para asegurar que cada persona cuente con los medios necesarios para llevar una vida genuinamente digna, consistente con dicha igualdad. Dado este punto de partida, el estándar de suficiencia no es sinónimo de un mínimo indispensable para no llevar una vida miserable, sino el estándar requerido para una igualdad moral de las personas. ${ }^{48}$

Por supuesto, dado que los recursos son limitados y la riqueza no se puede producir de la nada, alcanzar esta cantidad suficiente para cada individuo posiblemente requeriría importantes medidas redistributivas. Pero esto es enteramente contingente. La redistribución, para un igualitarista relacional, sería en tal caso instrumentalmente requerida por el valor de la igualdad, pero no sería parte constitutiva de su sentido. Esto implica que es perfectamente posible que una política orientada en su totalidad hacia el crecimiento económico logre de mejor manera el ideal de igualdad relacional, como algunos podrían argumentar. ${ }^{49}$ De esta manera, una de las divisiones más marcadas entre posturas políticas opuestas ( si acaso es preferible agrandar la torta que dividirla equitativamente, o viceversa) es, en la perspectiva del igualitarismo relacional, una cuestión netamente empírica y pragmática, cuya respuesta depende en definitiva de hechos sociales y económicos.

Al incorporar el interés en "hacer crecer la torta", y dada - como se verá- la relatividad de lo que cuenta como suficiente, la concepción suficientarista de la igualdad relacional es capaz de incorporar, bajo un

${ }^{48}$ Frankfurt, "Equality as a Moral Ideal", 38.

${ }^{49}$ Digo algunos debido a que un utilitarista puro —en el sentido de Parfit, esto es, alguien que cree que el único criterio moralmente importante es la cantidad total de utilidad, riqueza o bienestar en la sociedad - no argumentaría que enfocarse en el crecimiento es un buen medio para asegurar la igualdad social o para aliviar la pobreza, sino que ello constituye un fin moralmente valioso en sí mismo. Derek Parfit, "Equality and Priority", Ratio 10, n. ${ }^{\circ} 3$ (1997): 205. 
mismo ideal, nuestros diversos compromisos con la maximización de la riqueza, la justicia distributiva, la igualdad y — como se verá en segui$\mathrm{da}$ - la prioridad de aquellos que se encuentran en peores condiciones. ${ }^{50}$

Esta interpretación exigente, pluralista y flexible del suficientarismo, entendido desde la óptica de la igualdad relacional, permite además evitar las críticas tradicionalmente esbozadas contra el suficientarismo más estricto.

Una de esas críticas argumenta que, por sobre el umbral de suficiencia, el suficientarismo no tiene nada que decir: cualquier distribución de recursos o bienes sería adecuada o — si es que es posible juzgarlo- cuestionable sólo en base a razones no distributivas (por ejemplo, razones agregativas). ${ }^{51}$ Pero una concepción suficientarista como la que defiende este trabajo, que se entiende a sí misma como la consecuencia lógica del igualitarismo relacional —en lugar de en contraposición a él-, deja espacio para que existan implicancias distributivas de la igualdad distintas de la noción de suficiencia. El igualitarismo relacional simplemente da apoyo a la prioridad de la suficiencia como foco de la teoría y la acción políticas. Ello es perfectamente compatible con otras implicancias distributivas del igualitarismo, que tengan algo relevante que decir respecto a la distribución incluso más allá del umbral de suficiencia, pero que ocupan un lugar secundario frente a la prioridad — urgente en la mayoría de las sociedades contemporáneas - de la suficiencia.

Una segunda crítica usualmente esgrimida contra el suficientarismo es que éste no aportaría demasiado en términos normativos; después de todo, salvo por el libertario radical, nadie pareciera negar actualmente la necesidad de asegurar mínimos materiales para todos los ciudadanos. ${ }^{52}$ La respuesta a esta crítica está dada por lo ya señalado: el umbral de suficiencia no es la mera superación de la miseria o la indignidad. Se trata, por el contrario, de un estándar exigente: las condiciones materiales que evitan la existencia de relaciones basadas en la dominación, la opresión o la jerarquía de clase.

${ }^{50}$ No sigo pues, lo que Casal denomina la "tesis negativa", la cual plantea que sólo la suficiencia importa, y que la igualdad y la prioridad no tienen peso normativo alguno. Casal, "Por qué la suficiencia", 272-273.

${ }^{51}$ Shields, "The Prospects for Sufficientarianism", 103-4.

${ }^{52}$ Christian Schemmel, "Why Relational Egalitarians Should Care About Distributions", Social Theory and Practice 37, n. ${ }^{\circ} 3$ (2011): 369. En el mismo sentido, Casal, "Por qué la suficiencia", 272. 
Este criterio de suficiencia está ciertamente infraespecificado, y su determinación no es una cuestión evidente. ${ }^{53}$ No es claro qué nivel de suficiencia requiere el igualitarismo relacional. Pero esto no es problemático (a pesar de lo que sostienen algunos autores), ${ }^{54}$ sino que permite que lo que cuenta como suficiente dependa del contexto y, por sobre un umbral mínimo, éste sea parcialmente definido por la especificidad de cada tiempo y lugar, así como por el desarrollo tecnológico. Esta dependencia del contexto permite evitar la arbitrariedad de fijar el estándar de suficiencia en referencia a condiciones contingentes, pero, además, es consistente con su punto de partida relacional. Es el tipo de relaciones que se establecen entre las personas lo que define qué cuenta como suficiente.

Más aún, el carácter relativamente inespecífico del estándar de suficiencia le permite al igualitarismo relacional ser una poderosa arma de crítica normativa y de denuncia de la desigualdad manifiesta, que no requiere del desarrollo de una teoría completa de la justicia, pero que es suficiente para realizar la tarea de oponerse a las jerarquías sociales, la servidumbre y la opresión. ${ }^{55}$

El criterio de suficiencia es también relativo, en el sentido de que, aunque lo que cuenta como suficiente es lo mismo para todas las personas, sus circunstancias disímiles tienen un impacto en los recursos que cada una de ellas necesita para alcanzar el mismo umbral. No todos requieren, en definitiva, los mismos recursos. Por ejemplo, las personas que padecen impedimentos físicos o cognitivos pueden requerir recursos que las personas que no sufren tales impedimentos no requieren para alcanzar el mismo estándar de suficiencia. Esto es relevante, porque una concepción suficientarista de la igualdad, como la que defiende este trabajo, es perfectamente compatible con la idea de priorizar el bienestar de aquellos que se encuentran en peores condiciones. ${ }^{56}$ Ello vuelve a mostrar las virtudes de esta concepción pluralista y flexible de la igualdad relacional. 24.

${ }^{53}$ Como lo reconocía, tempranamente, Frankfurt, "Equality as a Moral Ideal",

${ }^{54}$ Schemmel, "Why Relational Egalitarians", 365.

${ }^{55}$ En este sentido, Wolff, "Social Equality and Social Inequality", 216.

${ }^{56}$ Respecto a la idea de prioridad, véase en general Parfit, "Equality and Priority". Respecto a la idoneidad del criterio de suficiencia para incorporar el atractivo intuitivo de la prioridad de los menos aventajados, véase Crisp, "Equality, Priority, and Compassion", 755-762. 


\section{DE LA IGUALDAD RELACIONAL A LA FILOSOFÍA POLIITICA DEL DERECHO PRIVADO57}

La igualdad relacional no tiene sólo implicancias relevantes en materia de filosofía política, como hemos visto en relación al suficientarismo. Ella también es relevante porque permite conectar la reflexión filosófica-política con instituciones jurídicas concretas y, en particular, con las del derecho privado. En efecto, una preocupación por la calidad de las relaciones que se establecen entre ciudadanos libres e iguales debería llevarnos también a redescubrir la relevancia de la justicia de las interacciones patrimoniales privadas entre personas, justicia que tradicionalmente ha sido designada como correctiva.

Un análisis exhaustivo de las implicancias del igualitarismo relacional para el derecho privado, en todo caso, excede las pretensiones de este trabajo. Por el contrario, aquí sólo se presenta el argumento que muestra la conexión entre ambas cuestiones, con objeto de fundamentar una exploración ulterior de dicha conexión y sugerir la orientación —más que el contenido- que esa exploración podría tener.

La conexión entre la justicia de las interacciones patrimoniales privadas y el ideal de la igualdad relacional no debería ser enteramente sorpresiva. El igualitarismo relacional está estrechamente vinculado a una concepción de la justificación política basada en la perspectiva de la segunda persona, de acuerdo a la cual las demandas de justicia son demandas relacionales que los agentes se formulan entre sí. ${ }^{58} \mathrm{La}$ estructura bipolar de las relaciones de derecho privado parece, en principio, un terreno estructuralmente fértil para la aplicación de criterios justificativos que asumen la perspectiva de la segunda persona. ${ }^{59} \mathrm{Al}$ mismo tiempo, y en términos sustantivos, la justicia en las relaciones de derecho privado es fundamentalmente la justicia correctiva. ${ }^{60} \mathrm{El}$ igualitarismo relacional, pues, es una orientación teórica cuyos supuestos son

${ }^{57}$ Esta sección del trabajo fue motivada por una conversación con Hanoch Dagan respecto a mi tesis doctoral.

${ }^{58}$ Anderson, "The Fundamental Disagreement", 3-6.

${ }^{59}$ Así lo han destacado, por ejemplo, Stephen Darwall \& Julian Darwall, "Civil Recourse as Mutual Accountability", en Morality, Authority, and Law: Essays in Second-Personal Ethics I (Oxford: Oxford University Press, 2013). En el mismo sentido, Andrew S. Gold, "A Moral Rights Theory of Private Law", William and Mary Law Review 52 (2011): 1896-1898.

${ }^{60}$ Ernest J. Weinrib, The Idea of Private Law (Oxford: Oxford University Press, 2012). 
consistentes con la estructura de las relaciones de derecho privado, las que a su vez se vinculan a la noción de justicia correctiva.

La noción de justicia correctiva es antigua, y por eso hablo de redescubrirla. ${ }^{61}$ Tal como ha sido tradicionalmente entendida, la justicia correctiva es la forma de justicia que "rectifica o remedia las desigualdades que surgen a partir de transacciones (...) entre individuos". ${ }^{62} \mathrm{En}$ el derecho privado, la justicia correctiva se asocia a la idea de que la responsabilidad civil rectifica la injusticia que una persona causa a otra. ${ }^{63}$

Cabe destacar que el redescubrimiento de la justicia correctiva y de la reflexión filosófico-política respecto al derecho privado no debiese interpretarse como asociado a una concepción no convencional, libertaria o preinstitucional del derecho privado. ${ }^{64}$ El derecho privado puede ser (correctamente, a mi juicio) concebido como un sistema de reglas enteramente convencionales y, por ejemplo, los derechos al cumplimiento contractual pueden ser concebidos como el mero resultado de la aplicación de esas reglas, producidas por instituciones políticas. Esa comprensión es plenamente consistente con el argumento presentado aquí. Para aceptar la tesis de que existen demandas de justicia aplicables a las interacciones entre privados no es necesario negar la convencionalidad del derecho privado. Es perfectamente posible decir que un sistema creado por convención, como el derecho privado, da lugar a cuestiones normativas de moralidad política que no se identifican necesariamente con la justicia distributiva.

En efecto, no toda la justicia es distributiva. Existen consideraciones de justicia que son estrictamente interpersonales, conmutativas o

${ }^{61}$ Para un análisis histórico de la distinción entre justicia correctiva y distributiva, véase Izhak Englard, Corrective and Distributive Justice: From Aristotle to Modern Times (Oxford: Oxford University Press, 2009).

${ }^{62}$ John Finnis, Natural Law and Natural Rights (Oxford: Clarendon Press, 1980), 178. La traducción es propia.

${ }^{63}$ Ernest J. Weinrib, "Corrective Justice in a Nutshell", The University of Toronto Law Journal 52, n. 4 (2002): 349.

${ }^{64}$ De esta manera, y contra lo que sostiene Beever, es perfectamente posible defender la relevancia de la justicia correctiva sin asumir un fundamento no convencional de los deberes del derecho privado. Se trata de dos cuestiones distintas. Allan Beever, Forgotten Justice: Forms of Justice in the History of Legal and Political Theory (Oxford: Oxford University Press, 2013). Una aguda defensa del convencionalismo respecto a las instituciones de derecho privado es la de Liam Murphy, "Private Law and Public Illusion. Lecture One: Artificial Morality" (2016), http://www.law.nyu.edu/sites/default/files/upload_documents/LectureOneArtificialMorality_\%20Murphy.pdf. 
correctivas, y que desempeñan un rol fundamental en el derecho privado. Es en las relaciones de derecho privado, precisamente, en las que se juega en parte importante la calidad de las relaciones cotidianas de las personas entre sí. Esto no implica, por cierto, que debamos ignorar la relevancia de consideraciones distributivas en el derecho privado, en particular si se tiene en cuenta que —al menos para una comprensión convencionalista del derecho privado - la evaluación de la justicia de la interacción entre individuos se hace contra el trasfondo de un contexto de (in)justicia distributiva. Desde el punto de vista de la moralidad política, pues, la dimensión interpersonal o conmutativa de la justicia es insuficiente. Esto es otra forma de decir que la justicia de las interacciones entre privados no se puede evaluar sin juicios (o asunciones) previos de carácter distributivo. ${ }^{65}$ Sin embargo, la tendencia contemporánea, al menos en la teoría política liberal igualitarista, parece ser precisamente la contraria: ignorar el hecho de que "la justicia del derecho privado no se agota en su dimensión de justicia distributiva". ${ }^{66}$

Por supuesto, existe una amplia literatura sobre la filosofía del derecho privado, que se refiere, entre otras cosas, a la justificación moral de las instituciones del derecho privado y al tipo de relaciones y deberes morales que surgen a partir de la interacción patrimonial entre los individuos. ${ }^{67}$ Sin embargo, esta literatura y la filosofía política con-

${ }^{65}$ Véase Wojciech Sadurski, "Social Justice and Legal Justice", Law and Philosophy 3, n. 3 (1984): 337.

${ }^{66}$ Martijn W. Hesselink, “Could a Fair Price Rule (or Its Absence) Be Unjust?”, European Review of Contract Law 11, n. 3 (2015): 190. La traducción es propia.

${ }^{67}$ Sólo por citar algunos ejemplos, véase Jules Coleman, Risks and Wrongs (Cambridge: Cambridge University Press, 1992); Peter Benson, "The Idea of a Public Basis of Justification for Contract", Osgoode Hall Law Journal 33 (1995): 273; Hanoch Dagan, "Pluralism and Perfectionism in Private Law", Columbia Law Review 112 (2012): 1.409; Melvin Eisenberg, "The Theory of Contracts", en The Theory of Contract Law, ed. Peter Benson (Cambridge: Cambridge University Press, 2001), 206-264; Charles Fried, Contract as Promise (Cambridge: Harvard University Press, 1981); Dori Kimel, From Promise to Contract: Towards a Liberal Theory of Contract (Oxford: Hart Publishing, 2003); Liam Murphy, "The Practice of Promise and Contract", en Philosophical Foundations of Contract Law, ed. Gregory Klass, Prince Saprai \& George Letsas (Oxford: Oxford University Press, 2014), 151-170; Arthur Ripstein, Equality, Responsibility, and the Law (Cambridge: Cambridge University Press, 1998); Arthur Ripstein, Private Wrongs (Cambridge: Harvard University Press, 2016); Stephen Smith, Contract Theory (Oxford: Oxford University Press, 2004); Seana Shiffrin, "The Divergence of Contract and Promise", Harvard Law Review 120, № 3 (2007): 708-753; Weinrib, The Idea of Private Law. 
temporánea de inspiración liberal igualitarista prácticamente no se han vinculado. O, mejor dicho, mientras la teoría del derecho privado suele mirar a la filosofía política, esta última suele ignorar a la primera, pese a la relevancia que - particularmente para una concepción relacional de la igualdad - tienen las relaciones de derecho privado. ${ }^{68}$

Por ello, aunque la evaluación de la justicia distributiva de las condiciones de trasfondo es necesaria para hacer un juicio integral acerca de la justicia de una situación, ${ }^{69}$ existen exigencias de justicia - tales como el deber de cumplir un contrato, de indemnizar en caso de incumplimiento o de compensar los daños causados por una negligenciaque, aun contra el trasfondo de la justicia distributiva, son de carácter relacional, y sólo pueden ser satisfechos por los individuos. ${ }^{70}$ Por supuesto, podemos imaginar maneras con las cuales podríamos colectivizar estas exigencias - por ejemplo, y como de hecho ocurre, a través de regímenes de seguro obligatorios en ciertos tipos de accidentes - ${ }^{71}$ De todas formas, bajo las condiciones actuales de la mayoría de los sistemas jurídicos occidentales, que si establecen sistemas de derecho privado y que no colectivizan los deberes que emanan de ellos, existe una pregunta - que ha sido consistentemente ignorada en la filosofía política contemporánea - respecto a cómo estos sistemas deberían diseñarse, de qué manera debiéramos entender y justificar los deberes que resultan de ellos, y qué tipo de exigencias morales ellos imponen a los individuos.

${ }^{68}$ Notando esta falta de atención hacia la teoría del derecho privado, ver Scheffler, "Distributive Justice".

${ }^{69} \mathrm{La}$ evaluación puede ser realizada también respecto al efecto del derecho privado en la justicia de trasfondo, sea desde una perspectiva distributivista fuerte - para la cual el derecho privado debe lograr la justicia distributiva- o débil - para la cual el derecho privado, al menos, no debe perjudicar la obtención de la justicia distributiva - Respecto a la distinción, véase Scheffler, "Distributive Justice".

${ }^{70}$ Hanoch Dagan \& Avihay Dorfman, "Just Relationships", Social Science Research Network (2014), 2-3, https://papers.ssrn.com/sol3/papers.cfm?abstract_ id $=2527970$.

${ }^{71}$ Aunque podría ser más difícil concebir una colectivización de los deberes contractuales, no existe ninguna razón lógica que la impida. Por ejemplo, como me ha sugerido Liam Murphy, una alternativa lógicamente posible - aunque probablemente poco práctica- sería el establecer un sistema en el cual los deudores que incumplen sean sancionados por el Estado con una multa, junto con una agencia estatal que le pague una indemnización al acreedor. 
Todas esas preguntas ofrecen un campo de análisis promisorio para la perspectiva del igualitarismo relacional. Más aún, la desatención respecto a estas cuestiones de justicia conmutativa, que suelen ser analizadas por los teóricos del derecho privado, es problemática para dicha perspectiva. En efecto, a menos que la preocupación respecto al carácter de las relaciones interpersonales en una sociedad sea un mero eslogan, quienes están convencidos de la relevancia de la igualdad relacional deberían centrar su atención en las relaciones interpersonales que de hecho se producen entre las personas. ${ }^{72}$ La igual dignidad y estatus de los ciudadanos es inexistente si, dentro de las relaciones privadas que marcan sus vidas, tales como — por ejemplo — el matrimonio, la relación laboral y el arriendo de una vivienda, ellos no pueden interactuar como iguales. Una parte cada vez más relevante de la vida de las personas y de sus expectativas de bienestar y reconocimiento depende, en las sociedades caracterizadas por el libre mercado, de este tipo de relaciones privadas. Tales relaciones, en definitiva, deben ser evaluadas desde el punto de vista de la justicia (no meramente distributiva). Para realizar tal evaluación, criterios horizontales acerca de la justicia de las interacciones entre privados, como los que provee el igualitarismo relacional, son fundamentales.

El impulso hacia el redescubrimiento de la justicia de las interacciones privadas no es relevante solamente para la filosofía política. También lo es para la teoría del derecho privado. Los teóricos del derecho privado llevan bastante tiempo discutiendo si - y en qué medidael derecho privado debiera ser interpretado o diseñado para facilitar el logro de objetivos de justicia distributiva. Muchos teóricos igualitaristas han argumentado en este sentido. ${ }^{73} \mathrm{Al}$ mismo tiempo, sin embargo, este argumento parece enfrentarse con dificultades insuperables en términos

${ }^{72}$ La mayoría de los teóricos del igualitarismo relacional se centran, al igual que sus pares igualitaristas de la suerte, en cuestiones tradicionalmente asociadas al derecho público: los derechos políticos, las libertades constitucionales, el sistema tributario, etcétera. Una de las pocas excepciones es el trabajo de Elizabeth Anderson en torno a las preguntas normativas generadas por los contratos de vientre subrogado (o vientres de alquiler). Al respecto, véase Elizabeth S. Anderson, "Is Women's Labor a Commodity?", Philosophy \& Public Affairs 19, n. ${ }^{\circ} 1$ (1990): 71 92; Anderson, Value in Ethics, cap. 8.

${ }^{73}$ El argumento clásico para el derecho de contratos es el de Anthony T. Kronman, "Contract Law and Distributive Justice", Yale Law Journal 89 (1980): 472. Véase también Aditi Bagchi, "Distributive Injustice and Private Law", Hastings Law Journal 60 (2008): 105. 
de atractivo político (en particular, el problema de imponer un costo excesivo a los individuos), ${ }^{74}$ factibilidad $^{75}$ y consistencia con las instituciones de derecho privado (como la falta de responsabilidad por omisión, o misfeasance, en el contexto del common law, ${ }^{76}$ y la estructura bipolar de las relaciones de derecho privado ${ }^{77}$ ). En este sentido, tal vez la igualdad relacional, que calza de mejor manera con esa estructura bipolar y con instituciones tradicionales del derecho privado, tales como la lesión enorme ${ }^{78}$ (basada en la idea de precio justo ${ }^{79}$ ) y la doctrina de unconscionability, ${ }^{80}$ puede ser una mejor herramienta en el intento por construir una teoría más igualitarista del derecho privado. Más aún, dado que estructural y sustantivamente, como hemos visto, la idea de igualdad relacional es consistente con las instituciones de derecho

${ }^{74}$ Véase, por ejemplo, Martín Hevia, Reasonableness and Responsibility: A Theory of Contract Law (Dordrecht: Springer, 2013); Arthur Ripstein, "The Division of Responsibility and the Law of Tort", Fordham Law Review 72 (2004): 1811.

${ }^{75}$ Este problema de factibilidad nace de que, como han argumentado varios autores, la adjudicación de derecho privado aparece como un ambiente institucional inadecuado para obtener fines de justicia distributiva, particularmente cuando se le compara al sistema tributario. Ver Louis Kaplow \& Steven Shavell, "Should Legal Rules Favor the Poor - Clarifying the Role of Legal Rules and the Income Tax in Redistributing Income", Journal of Legal Studies 29 (2000): 821.

${ }^{76}$ Peter Benson, "Misfeasance as an Organizing Normative Idea in Private Law", University of Toronto Law Journal 60, n. 3 (2010): 731-798.

${ }^{77} \mathrm{La}$ estructura bipolar del derecho privado consiste en que, típicamente, las relaciones jurídicas privadas y su proyección en el proceso judicial se producen entre dos partes con derechos y deberes correlativos. Weinrib, The Idea of Private Law, 114-44.

${ }^{78} \mathrm{La}$ lesión enorme es una institución jurídica que permite rescindir un contrato en caso de que exista una desproporción suficientemente significativa entre las contraprestaciones contractuales de las partes. Sobre la institución de la lesión enorme y su conexión con la igualdad en el intercambio, véase James Gordley, "Equality in Exchange", California Law Review 69, n. 6 (1981): 1587-1656.

${ }^{79}$ Respecto a la idea de precio justo, Hesselink, "Could a fair price rule (or its absence) be unjust?"; Andrea Perrone, "The Just Price Doctrine and Contemporary Contract Law: Some Introductory Remarks", Rivista Internazionale di Scienze Sociali 125, n. ${ }^{\circ} 2$ (2014): 217-236.

${ }^{80} \mathrm{La}$ doctrina de unconscionability en el common law permite a los jueces evaluar la justicia sustantiva de las cláusulas contractuales y, en su caso, negar la obligatoriedad del contrato en base a la excesiva injusticia, dureza o desproporción que éste genera. Respecto a esta doctrina, véase Charles L. Knapp, "Unconscionability in American Contract Law", en Commercial Contract Law: Transatlantic Perspectives, ed. Larry DiMatteo et al. (Cambridge: Cambridge University Press, 2013), 309-338. 
privado que ya tenemos, su introducción en la evaluación de tales instituciones no es disruptiva, sino que es coherente con los valores que, aunque implícitos, subyacen a ellas.

Por lo demás, el ideal de la igualdad relacional tiene implicancias relevantes respecto a cómo debiera ser determinado el contenido del derecho privado. Ello ocurre, por ejemplo, en el derecho de contratos. Como es sabido, ningún régimen de derecho privado otorga eficacia a todos los contratos. ${ }^{81}$ La igualdad relacional puede ser un criterio relevante en la determinación de cuándo otorgar o no tal eficacia a un contrato (por ejemplo, a través de la inclusión del estado de necesidad como un vicio del consentimiento ${ }^{82}$ o a través de la ineficacia de las cláusulas abusivas). ${ }^{83}$ Desde este punto de vista, sólo los contratos consistentes con la igualdad de las partes debieran ser obligatorios. Dado que el derecho de contratos es el soporte jurídico fundamental del intercambio de mercado, el ideal igualitario relacional se conecta también con la pregunta acerca de los límites del mercado, el cual - aunque es un mecanismo virtuoso de asignación de bienes y servicios en general- en ciertos casos puede degradar algunas formas de interacción humana, ${ }^{84}$ o puede "monetizar" tales interacciones. ${ }^{85}$ También en materia de derecho de contratos, el ideal de la igualdad relacional y la idea de no dominación a la que se asocia tienen importantes implicancias respecto a la pregunta acerca de cuándo un contrato debiera carecer de fuerza obligatoria por ser opresivo, ${ }^{86}$ aunque se haya accedido a él voluntariamente.

${ }^{81}$ Max Weber, Economy and Society: An Outline of Interpretive Sociology (Los Angeles: University of California Press, 1978), 668.

82 Así ocurre en la reciente reforma al derecho francés de obligaciones (introducida mediante la Ordenanza n. ${ }^{\circ}$ 2016-131), en la cual la invalidación, en todo caso, depende de que el estado de necesidad haya sido explotado.

${ }^{83}$ Como ocurre en el derecho chileno de protección al consumidor. Al respecto, véase Iñigo De la Maza, "Contratos por adhesión y cláusulas abusivas: ¿Por qué el Estado y no solamente el mercado?", Revista Chilena de Derecho Privado, n. ${ }^{\circ} 1$ (2003): 109-148.

${ }^{84}$ Allen E. Buchanan, Ethics, Efficiency, and the Market (Totowa: Rowman \& Littlefield, 1985), 101-103.

85 Albert Hirschman, The Passions and the Interests: Political Arguments for Capitalism Before Its Triumph (Princeton: Princeton University Press, 1997), 80.

${ }^{86}$ Jean Braucher, "Contract Versus Contractarianism: The Regulatory Role of Contract Law”, Washington and Lee Law Review 47 (1990): 719. 
Esto es sólo un ejemplo del tipo de preguntas que una consideración más atenta de la conexión entre el igualitarismo relacional y el derecho privado sugiere. Como se puede ver, la filosofía política de inspiración igualitarista haría bien en centrar su atención en las instituciones de derecho privado.

\section{CONCLUSIONES}

Este trabajo ha sugerido dos implicancias relevantes que la igualdad relacional, de estimarse plausible, tiene para la filosofía política. Una de esas implicancias consiste en que el ideal de igualdad relacional puede proveer sustento a una concepción suficientarista de la igualdad, la que permitiría reinterpretar de manera fértil y constructiva la división entre concepciones políticas opuestas, además de reorientar la preocupación de la teoría y la acción política igualitarista hacia asegurar las condiciones consistentes con la igualdad de las personas, en lugar de centrarse en la redistribución como tal. La segunda de estas implicancias es que el ideal de igualdad relacional debería llevarnos a redescubrir la justicia de las interacciones entre privados y, de esta manera, la filosofía política del derecho privado.

Como consecuencia de estas dos implicancias, quienes estamos comprometidos con alguna versión del igualitarismo relacional debiéramos avanzar, en el futuro, en la reflexión teórica acerca de estas cuestiones que han sido tradicionalmente abandonadas por el enfoque distributivista de la filosofía política contemporánea.

\section{BIBLIOGRAFÍA}

Alexander, Gregory S. "Pluralism and Property". Fordham Law Review 80 (2011): 1017-1052.

Anderson, Elizabeth. “¿Cuál es el punto de la igualdad?”. En Igualitarismo: Una discusión necesaria, editado por Javier Gallego \& Thomas Bullemore, traducido por Felipe Figueroa, 45-104. Santiago: Centro de Estudios Públicos, 2016.

_. "The Fundamental Disagreement between Luck Egalitarians and Relational Egalitarians". Canadian Journal of Philosophy 40 (2010): 1-23.

- Value in Ethics and Economics. Cambridge: Harvard University Press, 1995. 
“Is Women's Labor a Commodity?". Philosophy \& Public Affairs 19, n. ${ }^{\circ}$ (1990): 71-92.

Arneson, Richard J. "Democratic Equality and Relating as Equals". Canadian Journal of Philosophy 40 (2010): 25-52.

_. "Egalitarianism and the Undeserving Poor". Journal of Political Philosophy 5, n. ${ }^{\circ}$ (1997): 327-50.

Bagchi, Aditi. "Distributive Injustice and Private Law". Hastings Law Journal 60 (2008): 105-148.

Beever, Allan. Forgotten Justice: Forms of Justice in the History of Legal and Political Theory. Oxford: Oxford University Press, 2013.

Benson, Peter. "The Idea of a Public Basis of Justification for Contract". Osgoode Hall Law Journal 33 (1995): 273-336.

- "Misfeasance as an Organizing Normative Idea in Private Law". University of Toronto Law Journal 60, n. ${ }^{\circ} 3$ (2010): 731-798.

Berlin, Isaiah. Liberty. Editado por Henry Hardy. Oxford: Oxford University Press, 2002.

Braucher, Jean. "Contract Versus Contractarianism: The Regulatory Role of Contract Law". Washington and Lee Law Review 47 (1990): 697-739.

Buchanan, Allen E. Ethics, Efficiency, and the Market. Totowa: Rowman \& Littlefield, 1985.

Carter, Ian. "El respeto y la base de la igualdad". En Igualitarismo: Una discusión necesaria, editado por Javier Gallego \& Thomas Bullemore, traducido por Joaquín Vásquez, 193-228. Santiago: Centro de Estudios Públicos, 2016.

Casal, Paula. "Por qué la suficiencia no basta". En Igualitarismo: Una discusión necesaria, editado por Javier Gallego \& Thomas Bullemore, traducido por Javier Gallego, 269-301. Santiago: Centro de Estudios Públicos, 2016.

Cohen, G.A. "On the Currency of Egalitarian Justice". Ethics 99, n. ${ }^{\circ}$ (1989): 906-944.

Coleman, Jules. Risks and Wrongs. Cambridge: Cambridge University Press, 1992.

Contardo, Óscar. Siútico: Arribismo, abajismo y vida social en Chile. Santiago: Vergara, 2008.

Crisp, Roger. "Equality, Priority, and Compassion". Ethics 113, n. 4 (2003): 745763.

Dagan, Hanoch. "Pluralism and Perfectionism in Private Law". Columbia Law Review 112 (2012): 1409-1446.

Dagan, Hanoch \& Avihay Dorfman. "Just Relationships". Social Science Research Network (2014). https://papers.ssrn.com/sol3/papers.cfm?abstract $\mathrm{id}=2527970$.

Darwall, Stephen \& Julian Darwall. "Civil Recourse as Mutual Accountability". En Morality, Authority, and Law: Essays in Second-Personal Ethics I. Oxford: Oxford University Press, 2013.

De la Maza, Iñigo. "Contratos por adhesión y cláusulas abusivas: ¿Por qué el Estado y no solamente el mercado?". Revista Chilena De Derecho Privado 1 (2003): 109-148. 
Dworkin, Ronald. Sovereign Virtue: The Theory and Practice of Equality. Cambridge: Harvard University Press, 2002.

. "What is Equality? Part 2: Equality of Resources". Philosophy \& Public Affairs 10, n. ${ }^{\circ} 4$ (1981): 283-345.

Eisenberg, Melvin. "The Theory of Contracts". En The Theory of Contract Law, editado por Peter Benson, 206-264. Cambridge: Cambridge University Press, 2001.

Englard, Izhak. Corrective and Distributive Justice: From Aristotle to Modern Times. Oxford: Oxford University Press, 2009.

Finnis, John. Natural Law and Natural Rights. Oxford: Clarendon Press, 1980.

Frankfurt, Harry. "Equality and Respect". Social Research 64, n. ${ }^{0} 1$ (1997): 3-15. . "Equality as a Moral Ideal". Ethics 98, n. ${ }^{\circ} 1$ (1987): 21-43.

Fried, Charles. Contract as Promise. Cambridge: Harvard University Press, 1981.

Gallego, Javier \& Thomas Bullemore. "Introducción". En Igualitarismo: Una discusión necesaria, editado por Javier Gallego \& Thomas Bullemore, 15-41. Santiago: Centro de Estudios Públicos, 2016.

García Valverde, Facundo. "La prioridad del igualitarismo democrático". Revista de Filosofia 41, n. ${ }^{\circ} 1$ (2016): 79-96.

Gold, Andrew S. "A Moral Rights Theory of Private Law". William and Mary Law Review 52 (2011): 1873-1931.

Gordley, James. "Equality in Exchange". California Law Review 69, n. 6 (1981): 1587-1656.

Hesselink, Martijn W. "Could a Fair Price Rule (or Its Absence) Be Unjust?". European Review of Contract Law 11, n. ${ }^{\circ} 3$ (2015): 185-96.

Hevia, Martín. Reasonableness and Responsibility: A Theory of Contract Law. Dordrecht: Springer, 2013.

Hirschman, Albert. The Passions and the Interests: Political Arguments for Capitalism Before Its Triumph. Princeton: Princeton University Press, 1997.

Igneski, Violetta. "Equality, Sufficiency, and the State". Dialogue: Canadian Philosophical Review 46, n. ${ }^{\circ} 2$ (2007): 311-334.

Kaplow, Louis \& Steven Shavell. "Should Legal Rules Favor the Poor - Clarifying the Role of Legal Rules and the Income Tax in Redistributing Income". Journal of Legal Studies 29 (2000): 821-835.

Kimel, Dori. From Promise to Contract: Towards a Liberal Theory of Contract. Oxford: Hart Publishing, 2003.

Knapp, Charles L. "Unconscionability in American Contract Law". En Commercial Contract Law: Transatlantic Perspectives, editado por Larry DiMatteo, Qi Zhou, Severine Saintier \& Keith Rowley, 309-338. Cambridge: Cambridge University Press, 2013.

Knight, Carl. "Luck Egalitarianism”. Philosophy Compass 8, n. 10 (2013): 924-934.

Kronman, Anthony T. "Contract Law and Distributive Justice". Yale Law Journal 89 (1980): 472-511. 
Loewe, Daniel. "Refugiados climáticos: ¿Quién debe cargar los costos?”. Revista Interdisciplinar Da Mobilidade Humana 22, n. ${ }^{\circ} 43$ (2014): 169-187.

Lovett, Frank. "Republicanism". En The Stanford Encyclopedia of Philosophy, 2016. http://plato.stanford.edu/archives/spr2016/entries/republicanism/.

Miller, David. "Equality and Justice". Ratio 10, n. 3 (1997): 222-237.

Murphy, Liam. "The Practice of Promise and Contract". En Philosophical Foundations of Contract Law, editado por Gregory Klass, Prince Saprai \& George Letsas, 151-170. Oxford: Oxford University Press, 2014.

"Private Law and Public Illusion. Lecture One: Artificial Morality", 2016. http://www.law.nyu.edu/sites/default/files/upload_documents/ LectureOneArtificialMorality_\%20Murphy.pdf.

Nozick, Robert. Anarchy, State, and Utopia. New York: Blackwell, 1974.

Nussbaum, Martha C. Frontiers of Justice: Disability, Nationality, Species Membership. Cambridge: Harvard University Press, 2009.

Page, Olof. "Igualdad, suerte y responsabilidad". En Igualitarismo: Una discusión necesaria, editado por Javier Gallego \& Thomas Bullemore, 231-250. Santiago: Centro de Estudios Públicos, 2016.

Parfit, Derek. "Equality and Priority". Ratio 10, n. ${ }^{\circ} 3$ (1997): 202-221.

Perrone, Andrea. "The Just Price Doctrine and Contemporary Contract Law: Some Introductory Remarks". Rivista Internazionale di Scienze Sociali 125, n. ${ }^{\circ} 2$ (2014): 217-236.

Pettit, Philip. Republicanism. Oxford: Oxford University Press, 1999.

Rawls, John. A Theory of Justice. Cambridge: Harvard University Press, 1971.

Ripstein, Arthur. "The Division of Responsibility and the Law of Tort". Fordham Law Review 72 (2004): 1811-1844.

- Equality, Responsibility, and the Law. Cambridge: Cambridge University Press, 1998.

- Private Wrongs. Cambridge: Harvard University Press, 2016.

Sadurski, Wojciech. Equality and Legitimacy. Oxford: Oxford University Press, 2008.

. "Social Justice and Legal Justice". Law and Philosophy 3, n. 3 (1984): 329-354.

Scanlon, Thomas. "When Does Equality Matter?". 2005. https://www.law.yale.edu/ system/files/documents/pdf/Intellectual_Life/ltw-Scanlon.pdf.

Scheffler, Samuel. "Distributive Justice, the Basic Structure and the Place of Private Law". Oxford Journal of Legal Studies 35 (2015): 213-235.

- "Equality as the Virtue of Sovereigns: A Reply to Ronald Dworkin". Philosophy \& Public Affairs 31, n. 2 (2003): 199-206.

. "The Practice of Equality". En Social Equality, editado por Carina Fourie, Fabian Schuppert \& Ivo Wallimann-Helmer, 20-44. Oxford: Oxford University Press, 2015. 5-39. 
Schemmel, Christian. "Why Relational Egalitarians Should Care About Distributions". Social Theory and Practice 37, n. ${ }^{\circ} 3$ (2011): 365-390.

Shapiro, Ian. "On Non-domination". University of Toronto Law Journal 62, n. 3 (2012): 293-336.

Shields, Liam. "The Prospects for Sufficientarianism". Utilitas 24, n. 1 (2012): 101-117.

Shiffrin, Seana. "The Divergence of Contract and Promise". Harvard Law Review 120, n. ${ }^{\circ} 3$ (2007): 708-753.

."Egalitarianism, Choice-Sensitivity, and Accommodation". En Reason and Value: Themes from the Work of Joseph Raz, editado por Philip Pettit, 270302. Oxford: Oxford University Press, 2004.

Smith, Stephen. Contract Theory. Oxford: Oxford University Press, 2004.

Van Parijs, Philippe. "Why Surfers Should Be Fed: The Liberal Case for an Unconditional Basic Income". Philosophy \& Public Affairs 20, n. ${ }^{\circ} 2$ (1991): 101-131.

Weber, Max. Economy and Society: An Outline of Interpretive Sociology. Los Angeles: University of California Press, 1978.

Weinrib, Ernest J. "Corrective Justice in a Nutshell". The University of Toronto Law Journal 52, n. 4 (2002): 349-356.

- The Idea of Private Law. Oxford: Oxford University Press, 2012.

Wolff, Jonathan. "Social Equality and Social Inequality". En Social Equality, editado por Carina Fourie, Fabian Schuppert \& Ivo Wallimann-Helmer, 209225. Oxford: Oxford University Press, 2015. EP 
